\title{
Differences Between Current and Desired Physician Hypertension Management Roles Among Chinese American Seniors: a Qualitative Study
}

\author{
Emiley Chang, $M D^{1,2,3}$ (D) Victor Lin, $M D^{4}$, Regine Goh, $B S^{5}$, Canossa Chan, MEd ${ }^{6}$, \\ Michael K. Ong, MD' ${ }^{2,7}$, Edward Hui, $M D^{2}$, and Catherine Sarkisian, $M D^{2,8}$
}

\begin{abstract}
'Department of Medicine, Harbor-UCLA Medical Center, Los Angeles, CA, USA; ${ }^{2}$ Department of Medicine, David Geffen School of Medicine at University of California Los Angeles, Los Angeles, CA, USA; ${ }^{3}$ The Lundquist Institute, Torrance, CA, USA; ${ }^{4}$ Keck School of Medicine, University of Southern California, Los Angeles, CA, USA; ${ }^{5}$ Feinberg School of Medicine, Northwestern University, Chicago, IL, USA; ${ }^{6}$ American Cancer Society (Eastern Division), New York, NY, USA; ${ }^{D}$ Department of Medicine, Veterans Affairs Greater Los Angeles Healthcare System, Los Angeles, CA, USA; ${ }^{8}$ Geriatric Research Education and Clinical Center, Veterans Affairs Greater Los Angeles Healthcare System, Los Angeles, CA, USA.
\end{abstract}

BACKGROUND: Though patient-physician racial concordance correlates with better perceived shared decisionmaking, Chinese immigrants report low quality of care and have undertreated hypertension regardless of concordance.

OBJECTIVE: To inform efforts to change physician behavior and improve quality of hypertension care, we used role theory to explore differences between Chinese American seniors' descriptions of current and desired physician roles in hypertension management.

DESIGN: Qualitative interviews.

PARTICIPANTS: Immigrant Chinese Americans with hypertension age $\geq 65$ years in Los Angeles County.

APPROACH: We recruited 15 participants from a senior wellness center for language-matched interviews and blood pressure (BP) checks. Participants described current and desired physician activities for hypertension management. Bilingual research assistants translated audio recordings. Using thematic analysis, a threemember team independently reviewed and coded transcripts to identify themes regarding physician roles in hypertension management; discrepancies were discussed to achieve consensus. Themes were checked for validity in four subsequent focus groups.

RESULTS: We completed interviews in 2014. Interviewees' mean age was 70.6 years; seven were female and five had a systolic BP over $150 \mathrm{mmHg}$. All interviewees reported having race- and language-concordant primary care providers, were prescribed at least one BP medication, and had Medicare. Three major themes encompassed current and desired physician roles in hypertension management: technical expert, empathetic health steward, and health educator. Descriptions of current and desired physician roles differed for all themes,

Prior Presentations Results from this study were presented in poster format as "Physician lifestyle counseling among Chinese American seniors with hypertension" at the 2016 American Geriatrics Society Annual Scientific Meeting in Long Beach, CA.

Electronic supplementary material The online version of this article (https://doi.org/10.1007/s11606-020-06012-9) contains supplementary material, which is available to authorized users.

Received December 10, 2019

Accepted June 23, 2020

Published online September 21, 2020 most prominently for empathetic health steward and health educator. Participants desired but did not consistently experience interpersonal engagement or receive hypertension lifestyle counseling, citing visit time pressures. CONCLUSIONS: Among these Chinese American seniors, there remains a gap between current and desired physician roles in hypertension management, particularly interpersonal behaviors and education. Seniors deprioritized these roles in response to perceived physician role strain. Increased attention to the impact of perceived physician role strain might improve shared decision-making and hypertension management.

KEY WORDS: hypertension; Chinese Americans; lifestyle counseling; medication counseling; physician roles.

J Gen Intern Med 35(12):3471-7

DOI: $10.1007 / \mathrm{s} 11606-020-06012-9$

(C) Society of General Internal Medicine 2020

\section{INTRODUCTION}

Racial/ethnic concordance between patients and providers may be associated with greater satisfaction with care, patient-physician communication, patient engagement, and perceived shared decision-making. ${ }^{1-3}$ However, no relationship was found between racial/ethnic concordance and satisfaction with care among older Asian Americans, ${ }^{4}$ and Chinese-Canadian immigrants reported lower quality of care than European-Canadians regardless of concordance. ${ }^{5}$ Additionally, racial/ethnic concordance studies related to cardiovascular care have had negative or mixed findings for blood pressure (BP) control, medication adherence, and lifestyle counseling. ${ }^{6-8}$ To inform efforts to improve hypertension (HTN) management, our study compared current and desired physician roles in routine HTN management among immigrant Chinese American seniors, using symbolic interactionist role theory. In contrast to the structural-functional perspective, where roles are fixed and reinforced within society through learned responses, the symbolic interactionist view describes society as a framework in which individuals organize and 
interpret internal and external cues for action during engagement with others. ${ }^{9}$

\section{METHODS}

We conducted this qualitative exploratory study in preparation for a pilot clinical pharmacist program addressing potential gaps in routine physician HTN care among Chinese American seniors. We recruited 15 Chinese Americans aged 65 years and older to participate in individual semi-structured interviews and 42 to participate in one of four focus groups (FGs). All interview participants and 22 of the 42 FG participants were recruited through a Los Angeles County senior wellness center affiliated with a large independent physician association (IPA). The center director introduced the principal investigator (E.C.), a Chinese American physician, during the weekly lunch session announcements and displayed recruitment flyers. We recruited additional 22 FG participants through flyers at a senior services agency, local clinics, and local senior housing complexes, as well as announcements after community lectures at senior centers and a hospital.

Participants were eligible if they self-identified as Chinese American, were born outside of the USA, had a previous diagnosis of HTN, were prescribed antihypertensive medication, had a primary care physician, had health insurance, and could sit for an hour-long interview or a 1.5-h FG. Seniors were excluded if they did not meet all of the inclusion criteria, had a history of stroke, required help with medication administration, did not pass a 6 -item cognitive screening, ${ }^{10}$ or were enrolled in a HTN management program. Eligible seniors willing to participate in the interviews were interviewed in order of recruitment with attention to equal representation by gender. Remaining participants were assigned to FGs once interviews were completed.

A trained Chinese American community member (C.C.) fluent in both Cantonese and Mandarin conducted the language-matched interviews between February and March 2014 and FGs ( 2 in Mandarin and 2 in Cantonese) between March and April 2014. She completed a structured 6h FG facilitator training session led by UCLA research staff including techniques for eliciting input from all participants and how to follow the script including standard prompts. All interviews and 2 FGs were conducted in private rooms at the senior wellness center. To improve generalizability, 2 additional FGs were conducted at a senior services agency with participants recruited outside the senior wellness center. All participants provided written informed consent to participate and completed surveys in Chinese about their sociodemographic information, degree of English fluency, language concordance with their primary care physician, past medical history, medication routine, and use of traditional Chinese medicine. Participants also answered questions adapted from the 8-item Medication User Self-Evaluation (MUSE) tool to assess medication adherence issues. ${ }^{11}$
Interview participants completed the Short Form of the Taiwan Health Literacy Scale (STHLS). ${ }^{12}$ FG participants were asked country of origin. E.C. checked resting BP after survey completion. Participants received a $\$ 20$ gift card.

We conducted interviews and FGs in a two-stage process. We first conducted interviews to better understand the patient experience of HTN management and identify gaps in current care. During interviews, we asked participants to (1) describe physicians' current activities related to their HTN management; (2) describe additional desired physician activities; (3) recall what physicians told them about HTN management including BP medications; and (4) describe their personal behaviors related to HTN management. We then checked the validity of information obtained from the interviews by conducting FGs using a discussion guide. We provided FG participants a written summary of views expressed by two or more interviewees about physician activities in HTN management, read the summary aloud, and asked whether participants had similar or differing experiences (Appendix 1). We posted sheets listing reported barriers and facilitators to medication adherence and invited FG participants to explain, challenge, or add responses.

The facilitator took notes during each interview and FG. Immediately following each interview or FG, the principal investigator and facilitator reviewed the notes together and discussed emerging themes in order to refine questions and probes for subsequent interviews and FGs. We identified potential saturation of themes after 12 interviews and conducted 3 additional interviews to confirm. Bilingual Chinese American research assistants translated and transcribed interview and FG audio recordings into English (R.G., C.C.). Mandarin transcripts were compared with audio files and revised as needed (E.C., R.G.), with feedback provided to C.C. who accordingly revised the Cantonese transcripts.

We drafted a codebook using an inductive, exploratory approach. In contrast to a deductive approach seeking confirmation of a theory, an inductive exploratory approach views explanations as partial and subject to iterative revisions based on new angles of investigation. The codebook was reviewed by the study team and the codes were subsequently revised to incorporate feedback. Using thematic analysis, ${ }^{13}$ a threemember team (E.C., R.G., V.L.) independently reviewed and coded interview and FG transcripts to identify major themes regarding current and desired physician roles in HTN management. Major themes were those mentioned by at least 8 interviewees or participants in at least 3 FGs; within each major theme, we identified subthemes mentioned by at least 4 interviewees or participants in at least 2 FGs. Within each subtheme, we identified interviewees' examples of patientphysician tension arising from differences between current and desired physician roles and participants' responses to tension. The codebook was iteratively refined during the coding process, and the three-member team met weekly to discuss all discrepancies to achieve consensus. Quotations associated 
with each code were reviewed and formally organized into themes. We used the Atlas.ti 7.1.7 (Berlin) software program.

The study was approved by the UCLA institutional review board.

\section{RESULTS}

\section{Participant Characteristics}

Mean participant age was 71 years for interviews and 72 years for FGs. Seven (47\%) interview and 28 (66\%) FG participants were female. Six (40\%) interview and 19 (45\%) FG participants used Mandarin; 34 (81\%) FG participants were from China, Hong Kong, Macau, or Taiwan with the remainder from Southeast Asia (7) and Trinidad (1). Five (33\%) interview and $21(50 \%)$ FG participants had a resting systolic BP over $140 \mathrm{mmHg}$. While most (11 interview, $30 \mathrm{FG}$ ) reported checking their BP at home within the past week, half (2 interview, 6 FG) of those whose systolic BP was over 140 did not. All interviewees completed the STHLS easily without errors; therefore, we did not conduct it for the FG participants. Most participants were uncomfortable speaking English. Interviewees reported 10 unique primary care physicians (PCPs) and FG participants reported 28, with a total of 32 different PCPs. All interviewees had racially and linguistically concordant PCPs; all FG participants had racially concordant PCPs but 4 did not have linguistically concordant PCPs. All but one participant reported having medical insurance (Table 1).

\section{Major Themes}

In the interviews, we identified three major themes encompassing Chinese American seniors' views of current and desired physician roles in HTN management: technical expert, empathetic health steward, and health educator. Within each theme, we compared current and desired physician roles. FG participants confirmed the summary statement (Appendix 1) to be an accurate representation of the average experience of a senior with hypertension in the community, and elaborated on their experiences with physician medication prescribing and counseling. In this paper, we therefore primarily reported focus group feedback for the theme "Physician as Health Educator." Appendix 2 summarizes major themes and subthemes from the interviews, with quotes from interviews and FGs illustrating areas of tension between participants' views of current and desired physician roles.

\section{Physician as Technical Expert}

Participants perceived physicians primarily in a technical role focused on knowledge base, diagnosis, physical exam, test ordering, medication prescribing, and problem-solving (e.g., achieving desired health outcomes and fulfilling patient requests). Nearly all interviewees (13 of 15) mentioned

Table 1 Participant Characteristics

\begin{tabular}{|c|c|c|c|}
\hline & & Interviews & Focus groups \\
\hline Total number of participants & & 15 & 42 \\
\hline Average age in years & & 71 & 72 \\
\hline Female $(\%)$ & & $7(47 \%)$ & $28(66 \%)$ \\
\hline \multirow[t]{5}{*}{ Education } & Less than 8 th grade & $3(33 \%)$ & $11(26 \%)$ \\
\hline & Some high school & $4(27 \%)$ & $6(14 \%)$ \\
\hline & High school diploma or GED & $2(13 \%)$ & $9(21 \%)$ \\
\hline & Vocational school or some college & $2(13 \%)$ & $8(19 \%)$ \\
\hline & Bachelor's degree and above & $4(27 \%)$ & $8(19 \%)$ \\
\hline "Very" or "Mostly" comfortable speaking English & & $5(33 \%)$ & $3(7 \%)$ \\
\hline \multirow{3}{*}{ Health insurance (may endorse $\geq 1$ option) } & Medicare & $15(100 \%)$ & $40(95 \%)$ \\
\hline & Dual eligible & $4(27 \%)$ & $17(41 \%)$ \\
\hline & Private & $4(27 \%)$ & $4(10 \%)$ \\
\hline Financial strain in past 3 months & & $3(20 \%)$ & $11(26 \%)$ \\
\hline \multirow[t]{5}{*}{ Past medical history } & Diabetes & $6(40 \%)$ & $17(40 \%)$ \\
\hline & Heart problem & $6(40 \%)$ & $16(38 \%)$ \\
\hline & Kidney disease & $3(20 \%)$ & $6(14 \%)$ \\
\hline & High cholesterol & $8(53 \%)$ & $32(76 \%)$ \\
\hline & Ever smoked & $4(27 \%)$ & $5(12 \%)$ \\
\hline Checked home BP in past week & & $11(73 \%)$ & $30(71 \%)$ \\
\hline Physician speaks preferred language very well & & $15(100 \%)$ & $38(90 \%)$ \\
\hline \multirow{5}{*}{ TCM use within past 1 month } & Acupuncture & $3(20 \%)$ & $6(14 \%)$ \\
\hline & Herbal medication & $2(13 \%)$ & $1(2 \%)$ \\
\hline & Therapeutic massage & $3(20 \%)$ & $7(17 \%)$ \\
\hline & Tai chi and/or qi gong & $3(20 \%)$ & $9(21 \%)$ \\
\hline & None & $8(53 \%)$ & $24(57 \%)$ \\
\hline \multirow[t]{6}{*}{ MUSE items: It is easy to... } & Take medications on time & $14(93 \%)$ & $39(93 \%)$ \\
\hline & Understand instructions on medicine bottles & $15(100 \%)$ & $36(86 \%)$ \\
\hline & Get all the information needed about medicines & $10(67 \%)$ & $21(50 \%)$ \\
\hline & Set a schedule to take medicines daily & $15(100 \%)$ & $37(88 \%)$ \\
\hline & Learn how to take medications & $15(100 \%)$ & $36(86 \%)$ \\
\hline & Take medicines daily & $15(100 \%)$ & $33(79 \%)$ \\
\hline
\end{tabular}


medication prescribing as a physician role in HTN management. Six interviewees each reported other activities such as physical exam and achieving numeric or symptomatic resolution to their health problems, while five emphasized test ordering. In particular, the physician's ability to resolve or control health problems was seen as a marker of clinical skill. If a physician was unable to solve a problem, some interviewees felt it was appropriate to seek a second opinion or switch doctors. One interviewee stated, "[My doctor] could not control my blood pressure, therefore I have to change doctors."

Participants' descriptions of current physician roles generally matched desired roles within this theme. However, a few interviewees felt that the focus on technical clinical tasks may also detract from desired interpersonal or educational roles in the patient-physician relationship. One interviewee stated it was easier for busy physicians to prescribe a medication than to provide explanations: "That's all he can do, give some medicine. Nowadays doctors don't even explain much." Another interviewee stated she never really asked questions because her physician seemed focused on the examination: "I don't ask too many questions. He's always examining me."

\section{Physician as Empathetic Health Steward}

Participants described physicians as trustworthy and licensed authority figures who had assumed responsibility for their overall health. In exchange, the patient role was to follow instructions. One interviewee stated, "Whatever [my doctor] said, I should just follow his instructions. It's like the teacher who taught the student lessons. You have to do your homework. Otherwise why should you listen to the teacher?" At the same time, interviewees highly valued physicians who were caring, listened carefully and responded to patient concerns, individualized recommendations, and were easily accessible. One interviewee emphasized, "The other important thing is his attitude towards the patients. Is the doctor friendly? Is he sincere or not? Does he look down upon you?" The paternalistic physician role sometimes conflicted with participants' desires for more a more patient-centered decision-making style. One FG participant feared she would have fainted had she followed her physician's instructions to double her medication dose: "Therefore, you cannot just merely listen to your doctor. You need to analyze the situation yourself." Her statement elicited group laughter and one participant jokingly responded, "Then the doctors can close their doors." Participants appeared to resolve this internal tension and preserve external harmony by privately acting on their own. For example, one interviewee stressed the importance of trusting physicians and taking medications as prescribed: "You have to trust the doctor completely. Whatever medication he prescribes you just take it." Later in the interview, he revealed that he had self-discontinued his BP medication after he lost 40 pounds from cancer: "The doctor may think I am well because I am taking the medication. But I am the one stopping the medication." Interviewees with multiple physicians also described situations where they had compared physician responses to the same question: "I need to verify. Even though it may not look good, it's okay to have a second opinion. I trust these two doctors."

Participants consistently identified limited physician time as a major obstacle to desired interpersonal roles and accessibility. As a result, patients may need to initiate discussions for the physician to listen and respond to concerns. One interviewee advised, "Generally speaking, if you ask him, he will explain to you. He won't take the initiative to tell you." The topic of physician accessibility generated conflicted emotions. While a few interviewees expected physicians to have an emergency number or return calls, many relied on walk-in visits because they either did not expect or never received return calls, or preferred face-to-face encounters. Interviewees saw a busy practice as a successful practice, but also felt waiting was inconvenient. Two interviewees suggested a tradeoff between physician time engaged in interpersonal roles and patient wait times; one interviewee stated, "Yes, he's very patient and thorough but he has a lot of patients. He takes his time to see his patients and he explains thoroughly so often times I have to wait." Therefore, participants did not always expect physicians to fulfill desired interpersonal roles within current practice. One interviewee explained, "He will ask if you have any questions. I think that's enough, because so many people are waiting in line. I feel bad for taking too much of the doctor's time."

\section{Physician as Health Educator}

Lifestyle Modifications. When asked which HTN treatments physicians had discussed, interviewees readily identified medications but had more difficulty recalling advice on lifestyle modifications. Four mentioned reducing sodium intake; others recalled additional dietary advice such as reducing cholesterol intake and eating less meat. Two had been advised to reduce stress and avoid anger. Only one recalled being told to exercise more, and only one recalled being asked to lose weight. Five could not recall any specific physician advice; of these, three had never considered asking physicians about lifestyle modifications. To explain this wide variation in counseling patterns, one interviewee felt physicians had limited influence on diet and therefore limited responsibility: "It depends on yourself to control your diet. The doctor's job is prescribing medicine. He has done his job. He cannot control my diet." Three doubted being able to assess the effect of lifestyle changes on BP relative to medications. An interviewee recalled, "I asked the doctor if I could stop the medicine. He said yes but you need to change your lifestyle. Now, how do I know what I should change? If I don't take medication, [my BP will] be high. If I take medication, I don't understand where my change was." One interviewee pointed to time as a barrier to physician lifestyle 
counseling: "Do you think you have time to explain when you have 20 to 30 patients waiting out there?" On the other hand, interviewees indicated a strong desire for greater physician lifestyle counseling in HTN management. Nearly all (13 of 15) interviewees expressed interest in using lifestyle changes to help control BP. Six interviewees added that they preferred non-pharmacologic methods over medications. One participant shared, "There is a Chinese saying that all Chinese medications have $30 \%$ poison. [...] It's specifically pointing to Chinese medicine, but of course, both of them have it. [...] It's better not to take it if you don't need it." Four interviewees requested that physicians provide specific or individualized advice. According to one participant, "[Doctors] can tell us how we can better control our blood pressure such as exercising more and better diet. I will follow the suggestions $100 \%$, I'm very compliant. [...] If they give specific directions it's much more helpful, they know what's better for me."

In contrast to the limited information recalled from physician visits, participants shared detailed personal beliefs about nonpharmacologic HTN treatments, including physical activity, diet, and functional foods (Appendix 3). Participants tended to mention both low-sodium diet and low-fat diet as dietary modifications for HTN management. No one mentioned structured diet plans such as DASH. ${ }^{14}$ Seniors endorsed medicinal properties of foods such as Chinese celery or hawthorn, regardless of herbal use. Four participants mentioned sweating to reduce BP and two felt tempering mood changes was important. According to one participant, "I think exercise is important. Sweating is good for us, at least an hour of it. [...] Try to sleep better. Then, don't get too excited. Don't think too much. I get anxious too easily."

Medication Counseling. Participants viewed medication prescribing as a central physician role but described medication counseling as a limited role. Most did not recall receiving medication counseling beyond purpose, administration, and importance of medication adherence. Interviewees usually recalled more physician medication counseling if they had experienced an adverse reaction. Most interviewees could not recall receiving information on alternatives, potential side effects, mechanism, or interactions, but felt it would not change their decision to take a necessary medication. Trust in physician judgment appeared to be the most important factor in interviewees' decisions to take a new prescription medication. An interviewee who experienced stomach pain with a medication explained, "[My doctor] told me all medication have similar side effects. [...] Because I trust him and maybe this medicine is better, so I try the new medication." At the same time, the majority of interviewees desired information on side effects and welcomed more detailed medication counseling. However, they perceived physicians as too busy. One interviewee recalled, "I once asked [my doctor] and he said, 'The time I could spend on each patient is limited. I do not have enough time to explain to you the medication details." Only two interviewees felt that they would not understand such information; five interviewees reported reading medication information sheets on their own, albeit with difficulty due to limited English proficiency.

Participants in three FGs echoed this perception of time as a barrier to medication counseling and concern about side effects. In FG1, one participant emphasized that patients should engage their physicians: "I know they are busy but if you ask, they will explain. You have to ask. Then they will explain to you." Another participant agreed that it was important to ask questions about side effects, particularly due to the lack of medication information in Chinese. However, six other participants across all four FGs felt their physicians would not listen to them even if they raised concerns about potential side effects. In FG3, a participant stated he developed a persistent cough from his new BP medication; his physician merely prescribed a cough medicine and he ultimately changed doctors.

\section{DISCUSSION}

Our study is among the first to examine how patients with racially and linguistically concordant physicians respond to perceived differences between current and desired physician roles. Our results suggest that Chinese American seniors' cultural respect for authority, expressed desire to comply with health instructions, ${ }^{15}$ and strong interest in lifestyle methods of BP control might be leveraged in the difficult task of lifestyle behavior change. Chinese American participants viewed physicians as authorities with responsibility for their health ${ }^{16}$ and framed the patient-physician relationship as analogous to other social relationships with a hierarchical and moral dimension, such as parent-child and teacher-student. ${ }^{17}$ They also shared a rich cultural background with their physicians, such as proverbs about the close relationship between health, diet, and physical activity, as well as traditional Chinese medicine practices emphasizing the role of self-care (e.g., dietary changes including functional foods and structured movement therapies such as tai chi). ${ }^{18}$ However, seniors indicated that physicians failed to leverage this natural opportunity for lifestyle counseling due to clinic visit time pressures.

Role strain is an emotional state (e.g., anxiety, frustration) produced in response to problematic external conditions (stressors). ${ }^{9}$ Reported patient-physician tensions primarily arose from perceived physician role strain caused by role overload, defined as difficulty fulfilling role obligations due to limited time rather than ability. ${ }^{9}$ Tensions were addressed through role bargaining, a dynamic process where patients negotiate what would be considered adequate role performance by the physician. Chinese American seniors valued physicians with strong interpersonal skills, detailed explanations, and health education. However, given physician time constraints and concentration on the technical expert role, participants ultimately assumed that the roles of empathetic health steward and health educator were less essential in HTN management and changed physicians only when the physician 
failed to fulfill the technical role. Therefore, participants' lack of question-asking can be seen as an act of sensitivity, restraint, and deference rather than passivity. Our findings may have implications for national efforts promoting patient engagement and shared decision-making in health care: perceived physician role strain may need to be addressed before patients can comfortably engage in shared decision-making, even with racially and linguistically concordant providers. Perceived lack of physician time for education and counseling is a barrier that crosses language and culture.

In addition to advocating for policy change, physicians may begin to re-prioritize the health educator role by identifying opportunities for counseling and introducing patients to potential team members for more detailed counseling. As seniors see their primary care physicians more frequently than younger adults, brief lifestyle counseling could be delivered opportunistically (e.g., in response to lab results) and serially over time. Physicians may consider shared medical appointments to counsel multiple patients in longer visits. ${ }^{19}$ Physicians can also coordinate with bicultural health professionals embedded in community settings (e.g., independent pharmacies, senior centers, adult day health centers) to reinforce the value of lifestyle modifications for mutual patients with uncontrolled HTN, or collaborate with IPA- or HMO-employed nurses, pharmacists, or community health workers to provide services in these settings. Physicianpharmacist collaborations have been implemented in community-based medical offices to reduce BP among Latino and African American patients compared with usual care. ${ }^{20}$ Pharmacists have also been successfully co-located within black-owned barbershops to improve access to BP care. ${ }^{21}$ Other studies have used telehealth services to increase education and counseling between physician visits. ${ }^{22,} 23$

We tailored our clinical pharmacist HTN intervention based upon these study findings. Drawing upon the health steward role, we asked participating physicians to sign a letter inviting eligible patients to enroll in the program; during recruitment, we emphasized that the program would be conducted in their physicians' clinics and supervised by their physicians. We asked pharmacists to assist with the health educator role by prioritizing structured diet and lifestyle counseling and provided a 1-day motivational interviewing training. We also recruited bilingual and bicultural pharmacists to deliver the study intervention in collaboration with a pharmacy school.

\section{LIMITATIONS}

The majority of interviewees were recruited from a senior wellness center and may have a greater interest in education and counseling at baseline compared with seniors who did not attend the center. Findings may be specific to this IPA and may be difficult to disentangle from the ethnicity of the participants. We relied on patient recall of any physician advice rather than audio-recorded visits. Some participants went to the same PCP or specialists (e.g., nephrology), but due to small sample size we could not conclude whether some participants consistently recalled certain physicians providing more medication or lifestyle counseling compared with others. Many patients had been diagnosed with hypertension years earlier; thus, recall of any counseling given at diagnosis may not be accurately reflected. Similarly, the content and duration of counseling provided are unknown (e.g., medication side effects, what constitutes a low-sodium diet). Regarding lack of weight loss counseling recalled by patients, we did not have patient weights to assess appropriateness. Finally, our small sample size limits our analysis to range and general frequency of themes.

\section{CONCLUSION}

Immigrants and their children currently comprise $28 \%$ of the US population and the proportion continues to grow. ${ }^{24}$ With immigration from Asia outpacing Latin America since 2010, Asians are projected to become the largest immigrant group in the USA by $2055,{ }^{25}$ and the proportion of Asian Americans aged 65 and older will double by $2060 .{ }^{26}$ Our communitypartnered study with bicultural investigators could serve as a model to better understand the cultural context of health care among other immigrant communities. Our study contributes to the literature on patient-physician communication by comparing Chinese American patients' views of current and desired physician roles, focusing on areas of difference. Despite having racially and linguistically concordant physicians, immigrant Chinese American seniors with HTN desired but did not expect education and counseling to be a high-priority physician role due to perceived physician time pressures, nor did they recall routinely receiving structured medication or lifestyle counseling.

Future studies may investigate potential associations between perceived physician role strain and patient satisfaction with physician care within different racial/ethnic minority communities, as well as the feasibility and acceptability of providing telehealth services or shared medical appointments led by non-physician team members. Decreasing perceived physician role strain might be an important lever to improve shared decision-making and HTN management.

Acknowledgments: We are grateful to the staff at the senior wellness center for allowing us to recruit and interview study participants at the center.

Corresponding Author: Emiley Chang, MD; Department of Medicine, Harbor-UCLA Medical Center, Los Angeles, CA, USA (e-mail: emiley.chang@lundquist.org).

Funding This work was supported by the UCLA/Hartford Center of Excellence; UCLA NRSA General Internal Medicine Fellowship in Primary Care and Health Services Research; Los Angeles Stroke Prevention/Intervention Research Program (SPIRP) in Health Disparities Fellowship; UCLA Faculty Training Program in Geriatric Medicine, Psychiatry and Dentistry; the National Institute on Aging Igrant number 1K24AG047899-01 to Dr. Sarkisian]; UCLA Clinical and Translational Science Institute (NIH/NCATS Grant \# UL1TR001881); 
NIA Resource Centers for Minority Aging Research IV / Center for Health Improvement of Minority Elderly III. The funders were not involved in the study design; the collection, analysis, and interpretation of data; the writing of the report; nor the decision to submit the article for publication.

\section{Compliance with Ethical Standards:}

All participants provided written informed consent to participate. The study was approved by the UCLA institutional review board. We confirm all personal identifiers have been removed or disguised so the quoted participants are not identifiable and cannot be identified through the details of the manuscript.

Conflict of Interest: The authors declare that they do not have a conflict of interest.

\section{REFERENCES}

1. Laveist RA, Nuru-Jeter A. Is doctor-patient race concordance associated with greater satisfaction with care? J Health Soc Behav. 2002;43:296306.

2. Cooper-Patrick L, Gallo JJ, Gonzales JJ, et al. Race, gender, and partnership in the patient-physician relationship. J Am Med Assoc. 1999;282:583-9.

3. Cooper LA, Roter DL, Johnson RL, Ford DE, Steinwachs DM, Powe NR. Patient-centered communication, ratings of care, and concordance of patient and physician race. Ann Intern Med. 2003;139:907-15.

4. Phillips KL, Chiriboga DA, Jang Y. Satisfaction with care: the role of patient-provider racial/ethnic concordance and interpersonal sensitivity. J Aging Health. 2012;24(7):1079-90.

5. Waibel S, Wong ST, Katz A, Levesque JF, Nibber R, Haggerty J. The influence of patient-clinician ethnocultural and language concordance on continuity and quality of care: a cross-sectional analysis. CMAJ Open. 2018;6(3):E276-E284.

6. Schoenthaler A, Montague E, Baier Manwell L, Brown R, Schwart MD, Linzer M. Patient-physician racial/ethnic concordance and blood pressure control: the role of trust and medication adherence. Ethn Health. 2014;19:565-78. https://doi.org/10.1080/13557858.2013. 857764.

7. Traylor AH, Schmittdiel JA, Uratsu CS, Mangione CM, Subramanian U. Adherence to cardiovascular disease medications: does patientprovider race/ethnicity and language concordance matter? J Gen Intern Med. 2010;25:1172-7. https://doi.org/10.1007/s11606-010-1424-8.

8. Eamranond PP, Davis RB, Phillips RS, Wee CC. Patient-physician language concordance and lifestyle counseling among Spanish-speaking patients. J Immigr Minor Health. 2009;11:494-8. https://doi.org/10. 1007/s 10903-008-9222-7.

9. Hardy ME, Conway ME. Role Theory: Perspectives for Health Professionals. Norwalk: Appleton \& Lange; 1988.

10. Callahan CM, Unverzagt FW, Hui SL, Perkins AJ, Hendrie HC. Six-item screener to identify cognitive impairment among potential subjects for clinical research. Med Care. 2002;40:771-81.

11. Doucette WR, Chang EH, Pendergast JF, Wright KB, Chrischilles EA, Farris KB. Development and initial assessment of the medication user self-evaluation (MUSE) tool. Clin Ther. 2013;35:344-50. https://doi.org/ 10.1016/j.clinthera.2013.02.010
12. Pan FC. A Short Form of the Taiwan Health Literacy Scale (THLS) for Chinese-Speaking Adults. Int Schol Sci Res Innov. 2010;4:1054-1059.

13. Braun V, Clarke V. Using thematic analysis in psychology. Qual Res Psychol. 2006;3:77-101. https://doi.org/10.1191/1478088706qp063oa.

14. Appel LJ, Moore TJ, Obarzanek E, et al. A clinical trial of the effects of dietary patterns on blood pressure. N Engl J Med 1997;336:1117-24.

15. Wang J, Matthews JT. Chronic disease self-management: Views among older adults of Chinese descent. Geriatr Nurs. 2010;31:86-94. https:// doi.org/10.1016/j.gerinurse.2009.11.001.

16. Wang JH, Adams IF, Pasick RJ, et al. Perceptions, expectations, and attitudes about communication with physicians among Chinese American and non-Hispanic white women with early stage breast cancer Support Care Cancer. 2013;21:3315-25. https://doi.org/10.1007/ s00520-013-1902-8

17. Rodwin MA. Strains in the fiduciary metaphor: divided physician loyalties and obligations in a changing health care system. Am J Law Med. 1995;21:241-57.

18. Weng $\mathbf{W}$, Chen $\mathbf{J}$. The eastern perspective on functional foods based on traditional Chinese medicine. Nutr Rev. 1996;54:S11-6.

19. Hayhoe B, Verma A, Kumar S. Shared medical appointments. BMJ. 2017;358:j4034. https://doi.org/10.1136/bmj.j4034.

20. Carter BL, Coffey CS, Ardery G, et al. Cluster-randomized trial of a physician/pharmacist collaborative model to improve blood pressure control. Circ Cardiovasc Qual Outcomes. 2015;8(3):235-43. https://doi. org/10.1161/CIRCOUTCOMES.114.001283.

21. Victor RG, Blyler CA, Li N, et al. Sustainability of Blood Pressure Reduction in Black Barbershops. Circulation. 2019;139(1):10-19. https://doi.org/10.1161/CIRCULATIONAHA.118.038165.

22. Jackson GL, Oddone EZ, Olsen MK, et al. Racial differences in the effect of a telephone-delivered hypertension disease management program. J Gen Intern Med. 2012;27(12):1682-9. https://doi.org/10.1007/s11606012-2138-x.

23. Niznik JD, He H, Kane-Gill SL. Impact of clinical pharmacist services delivered via telemedicine in the outpatient or ambulatory care setting: A systematic review. Res Soc Adm Pharm. 2018;14(8):707-717. https://doi. org/10.1016/j.sapharm.2017.10.011.

24. Batalova J, Blizzard B, Bolter J. Migration Policy Institute. Frequently Requested Statistics on Immigrants and Immigration in the United States. Published Feb 14, 2020. Available at https://www. migrationpolicy.org/article/frequently-requested-statistics-immigrantsand-immigration-united-states. Accessed March 20, 2020.

25. Radford J. Pew Research Center. Key findings about U.S. immigrants. Published June 17, 2019. Available at https://www.pewresearch.org/ fact-tank/2019/06/17/key-findings-about-u-s-immigrants/. Accessed March 20, 2020

26. Administration for Community Living, U.S. Department of Health and Human Services. 2018 Profile of Asian Americans Age 65 and Over. Published Oct 2019. Available at https://acl.gov/sites/default/files/ Aging and Disability in America/2018AsA_OAProfile.pdf. Accessed March 20, 2020

Publisher's Note Springer Nature remains neutral with regard to jurisdictional claims in published maps and institutional affiliations. 